\title{
A NOTE ON INJECTIVE MODULES OVER A d.g. NEAR-RING
}

\author{
A. OSWALD
}

In [3] an attempt was made at proving the following result:

(A) An $N$-module $M$ over a d.g. near-ring is injective if and only if for each right ideal $u$ of $N$ and each $N$-homomorphism $f: u \rightarrow M$ there exists an element $m \in M$ with $f(a)=m a$ for all $a \in u$.

In this note we present two examples. The first is a counterexample to (A) and the second illustrates one point at which the attempt made in [3] fails. As in [3] our near-rings are left near-rings (i.e. $a(b+c)=a b+a c$ ) with multiplicative identities. We recall that a near-ring $N$ is distributively generated (d.g.) if there is a multiplicative subsemigroup $S$ of $N$ with $\left(n_{1}+n_{2}\right) s=n_{1} s+n_{2} s$ for all $n_{1}, n_{2} \in N, s \in S$, and $N$ is generated additively by $\{\sigma: \sigma \in S$ or $-\sigma \in S\}$. In this way we can regard the ring $Z$ of integers as a near-ring generated by $\{1\}$. To indicate that we are doing this we write $(Z, 1)$ for the d.g. near-ring of integers. All groups can now be considered as $(Z, 1)$-modules. (See [1] for further details).

Clearly (A) is equivalent to

(B) An $N$-module $M$ over a d.g. near-ring $N$ is injective if and only if for each right ideal $u$ of $N$ and each $N$-homomorphism $f: u \rightarrow M$ there is an $N$-homomorphism $\phi: N \rightarrow M$ such that $\phi$ restricted to $u$ is $f$.

We write all our groups additively (without implying commutativity) and then recall that a group $G$ is divisible if and only if for each $a \in G$ and each positive integer $n$ there is an element $b \in G$ with $n b=a$.

LEMMA. If (B) is true then a group $G$ is divisible if and only if it is an injective $(Z, 1)$-module.

Proof. This is a straightforward generalisation of the corresponding result for rings (See e.g. [2; p. 51]).

In particular, if (B) is true then $Q$, the group of rational numbers, is an injective $(Z, 1)$-module.

If $M$ is any set we let $\operatorname{Sym}(M)$ be the symmetric group on $M$. For $f \in \operatorname{Sym}(M)$ let $S(f)=\{m \in M: m f \neq m\}$. If $A$ is any infinite cardinal then $\operatorname{Sym}(M, A)=$ $\{f \in \operatorname{Sym}(M):|S(f)|<A\}$. We also denote by $A^{+}$the next larger cardinal after

Received by the editors July 14, 1976. 
$A$. Let $B$ be any infinite cardinal with $B>|Q|^{+}$and $M$ be any set with $|M|=B$. Then by $[4 ; 11.5 .4$.] $Q$ can be imbedded in the simple group $S=$ $\operatorname{Sym}\left(M, B^{+}\right) / \operatorname{Sym}(M, B)$ and by $[4,11.5 .9],.\left[\operatorname{Sym}\left(M, B^{+}\right): \operatorname{Sym}(M, B)\right]>B>$ $|Q|$. But $Q$ is $(Z, 1)$-injective so the diagram<smiles>O=[V](=O)(O)S</smiles>

where $\alpha$ is the imbedding and $i$ the identity on $Q$ can be completed to the commutative diagram<smiles></smiles>

However, since $\operatorname{ker}(\beta)$ is a normal subgroup of $S$ we get $\operatorname{ker}(\beta)=0$ from which $\beta$ is $1-1$ which contradicts the cardinalities or $\operatorname{ker}(\beta)=S$ which contradicts $\beta$ being an extension of $i$. It follows that (B) is false.

Of course (B) is only false in the "if" part. This is also true of (A). In [3] consideration is given to the diagram

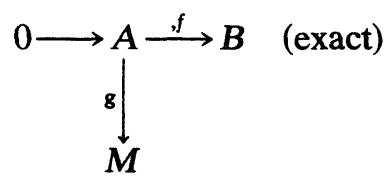

where $A, B, M$ are $N$-modules, and we write $C=f(A) \subset B$. The submodule $\bar{C}$ $B$ generated by $C$ is

$$
\bar{C}=\left\{\sum_{i=1}^{n}\left(-b_{i}+f\left(a_{i}\right)+b_{i}\right): b_{i} \in B, a_{i} \in A\right\} .
$$

A mapping $h: \bar{C} \rightarrow M$ is introduced and defined by

$$
h\left(\sum\left(-b_{i}+f\left(a_{i}\right)+b_{i}\right)\right)=\sum h\left(f\left(a_{i}\right)\right)
$$

where $h: C \rightarrow M$ is defined by $h(f(a))=g(a)$. It is then asserted that $h$ restricted to $C$ is $h$ but this is not necessarily true.

Again take $N=(Z, 1)$ and $A=B=M$ to be non-abelian groups regarded as $(Z, 1)$-modules. Let $f, g$ be the identity. For $a, a_{1} \in A$

$$
h\left(f\left(-a_{1}\right)+f(a)+f\left(a_{1}\right)\right)=h(f(a))=a \quad\left(\text { by }(i), \text { since } f\left(a_{1}\right) \in B\right)
$$

However,

$$
h\left(f\left(-a_{1}\right)+f(a)+f\left(a_{1}\right)\right)=h\left(f\left(-a_{1}+a+a_{1}\right)\right)=h\left(f\left(-a_{1}+a+a_{1}\right)\right)=-a_{1}+a+a_{1}
$$


and we deduce that $a_{1}+a=a+a_{1}$ for all $a, a_{1} \in A$ which is false. It is now impossible to establish that $h$ is well defined.

\section{BIBLIOGRAPHY}

1. A. Fröhlich, Distributively Generated near-rings (II Representation Theory) Proc. L.M.S. (3) (8) (1958), 95-108.

2. J. P. Jans, Rings and Homology, Holt, Rinehart and Winston, 1964.

3. V. Seth and K. Tewari, On injective near-ring modules, Canad. Math. Bull. Vol. 17 (1974), 137-141.

4. W. R. Scott, Group Theory, Englewood Cliffs N.J.: Prentice-Hall, 1964.

Department of Mathematics and Statistics,

TeEside Polytechnic,

MiDDLESBROUGH,

Cleveland,

U.K. 